\title{
AJK2011-04009
}

\section{AN ADAPTIVE MOVING-MESH RELAXATION SCHEME FOR COMPRESSIBLE TWO-PHASE BAROTROPIC FLOW WITH CAVITATION}

\author{
Keh-Ming Shyue \\ Department of Mathematics \\ National Taiwan University \\ Taipei, Taiwan 10617 \\ Email: shyue@ntu.edu.tw
}

\begin{abstract}
We describe a simple relaxation scheme for the efficient numerical resolution of compressible two-phase barotropic flow with and without cavitation on moving meshes. The algorithm uses a curvilinear-coordinate formulation of the relaxation model proposed by Saurel et al. (J. Comput. Phys. 228 (2009) 1678-1712) as the basis, and employs a wave-propagation based relaxed scheme to solve the model system on a mapped grid that is constructed by a conventional mesh-redistribution procedure for better solution adaptation. Sample numerical results in both one and two space dimensions are present that show the feasibility of the proposed method for practical problems.
\end{abstract}

\section{NOMENCLATURE}

$\alpha_{k} \quad$ Volume fraction for the fluid phase $k$.

$Y_{k}=\rho_{k} / \rho \quad$ Mass fraction for the fluid phase $k$.

$\rho_{k} \quad$ Density for the fluid phase $k$.

$p_{k} \quad$ Pressure for the fluid phase $k$.

$c_{k} \quad$ Speed of sound for the fluid phase $k$.

$\rho=\sum_{k=1}^{2} \alpha_{k} \rho_{k} \quad$ Total density.

$p=\sum_{k=1}^{2} \alpha_{k} p_{k} \quad$ Total pressure.

$u_{i} \quad$ Particle velocity in the $x_{i}$-direction.

$U_{j}=\sum_{i=1}^{N_{d}} u_{i} J_{j i} \quad$ Contravariant velocity in the $\xi_{j}$-direction.

c Mixture speed of sound.

$c_{f}$ Frozen speed of sound.

$\mu$ Relaxation parameter.

$Q_{i j}^{n} \quad$ Approximate solution of the cell average in $C_{i j}$ at time $t_{n}$.

$\Delta \xi_{i} \quad$ Mesh size in the $\xi_{i}$-direction.
$\Delta t \quad$ Time step from the current time $t_{n}$ to the next $t_{n+1}$. $\kappa_{i j}=J\left(C_{i j}\right) \quad$ Jacobian of grid mapping for cell $C_{i j}$.

$M_{w} \quad$ Total number of waves.

\section{INTRODUCTION}

Cavitation is commonly defined as a phenomenon in a liquid-flowing system when the pressure of the liquid falls sufficiently low in some region of the flow so that vapor bubbles are formed. The study of the dynamics of cavitation is an active research in many fields of science and engineering. Typical examples in relation to various features and characteristics of cavitating flows can be found in [1-3], for example.

To compute cavitating flow numerically, one popular approach among them is to use a two-phase barotropic model (cf. [4]) in that, if we ignore the physical effects such as mass transfer, surface tension, and viscosity, the Eulerian formulation of the basic conservation laws in $N_{d} \geq 1$ space dimension takes the form

$$
\frac{\partial}{\partial t}\left(\begin{array}{c}
\alpha_{1} \rho_{1} \\
\alpha_{2} \rho_{2} \\
\rho u_{i}
\end{array}\right)+\sum_{j=1}^{N_{d}} \frac{\partial}{\partial x_{j}}\left(\begin{array}{c}
\alpha_{1} \rho_{1} u_{j} \\
\alpha_{2} \rho_{2} u_{j} \\
\rho u_{i} u_{j}+p(\rho) \delta_{i j}
\end{array}\right)=0
$$

for $i=1,2, \ldots, N_{d}$. To close the system, the phasic pressure $p_{k}(\rho)$ for $k=1,2$ is assumed to be a one-to-one function of the density (this should be true at least locally), and so we may use the saturation condition $\alpha_{1}+\alpha_{2}=1$ directly, yielding a nonlinear 
algebraic equation of the form

$$
g(p)=\frac{\alpha_{1} \rho_{1}}{\rho_{1}(p)}+\frac{\alpha_{2} \rho_{2}}{\rho_{2}(p)}-1=0
$$

to be solved for the pressure $p$, where the quantities $\alpha_{1} \rho_{1}$ and $\alpha_{2} \rho_{2}$ are known a priori. With that, it is easy to find the remaining flow variables such as $\rho_{1}, \rho_{2}, \alpha_{1}$, and $\alpha_{2}$.

In this case, it is known that combining (1) with (2) gives a hyperbolic model that is viable for a class of homogeneous two-phase barotropic flow problems with and without cavitation. However, due to the non-monotonic behavior of the mixture sound speed (denoted by $c$ ) versus the volume fraction, $1 / \rho c^{2}=$ $\alpha_{1} / \rho_{1} c_{1}^{2}+\alpha_{2} / \rho_{2} c_{2}^{2}$, in the two-phase coexistent regions, it poses a major difficulty to attain a suitable stability condition when the model is discretized by a diffuse-interface method explicitly.

To overcome this numerical difficulty, we are interested in a relaxation approach proposed by Saurel et al. [5] in that in addition to (1) a transport equation with a stiff relaxation source term is included in the model for the volume fraction, such as $\alpha_{1}$, of the form

$$
\frac{\partial \alpha_{1}}{\partial t}+\sum_{j=1}^{N_{d}} u_{j} \frac{\partial \alpha_{1}}{\partial x_{j}}=\mu\left(p_{1}\left(\rho_{1}\right)-p_{2}\left(\rho_{2}\right)\right)
$$

In contrast with the aforementioned conventional model that makes use of the saturation condition (2), here the equilibrium pressure $p$ is obtained by taking the limit of infinite relaxation $\mu \rightarrow \infty$ to the solution of (3), yielding $p=p_{1}\left(\rho_{1}\right)=p_{2}\left(\rho_{2}\right)$, and so an algebraic equation for the relaxed volume fraction $\alpha_{1}$,

$$
\hat{g}\left(\alpha_{1}\right)=p_{1}\left(\frac{\alpha_{1} \rho_{1}}{\alpha_{1}}\right)-p_{2}\left(\frac{\alpha_{2} \rho_{2}}{1-\alpha_{1}}\right)=0 .
$$

It is important to note that since this relaxation model will be solved by a fractional-step method in the zero relaxation limit $\mu \rightarrow 0$, it possesses a nice monotonic behavior of the frozen speed of sound versus the mass fractions, $c_{f}^{2}=Y_{1} c_{1}^{2}+Y_{2} c_{2}^{2}$, and so is an easier one to use as compared to the above conventional model for numerical approximation.

It is worthwhile to mention that the single-phase barotropic flow model devised in $[6,7]$ works well for isentropic cavitating problems, but is not suitable for general non-cavitating twophase problems. This is unlike the fluid-mixture model proposed by the author [8] which works quite well for the two-phase barotropic scenario, but has experienced numerical difficulties for problems with cavitation.

Our goal in this work is to employ a state-of-the-art adaptive moving-mesh method (cf. [9]) for the efficient numerical resolution of compressible two-phase barotropic flow with and without cavitation in general non-rectangular domains. This is a fundamental step in our further development of the method to more complicated cavitating flows of practical importance (cf. [5, 10, 11]).

\section{MODEL EQUATIONS IN CURVILINEAR COORDINATES}

We begin our discussion by introducing a coordinate mapping from the physical domain $\left(x_{1}, x_{2}, x_{3}\right)$ in three dimensions $N_{d}=3$ to the computational domain $\left(\xi_{1}, \xi_{2}, \xi_{3}\right)$ via the relations

$$
\begin{aligned}
& d x_{1}=a_{1} d \xi_{1}+a_{2} d \xi_{2}+a_{3} d \xi_{3}, \\
& d x_{2}=b_{1} d \xi_{1}+b_{2} d \xi_{2}+b_{3} d \xi_{3}, \\
& d x_{3}=c_{1} d \xi_{1}+c_{2} d \xi_{2}+c_{3} d \xi_{3},
\end{aligned}
$$

where $a_{i}, b_{i}, c_{i}$ for $i=1,2,3$ are the metric terms of the mapping. Then under this mapping, the relaxation model described above can be transformed into the new coordinate system as

$$
\begin{aligned}
& \frac{\partial}{\partial t}\left(\alpha_{1} \rho_{1}\right)+\frac{1}{J} \sum_{j=1}^{N_{d}} \frac{\partial}{\partial \xi_{j}}\left(\alpha_{1} \rho_{1} U_{j}\right)=0, \\
& \frac{\partial}{\partial t}\left(\alpha_{2} \rho_{2}\right)+\frac{1}{J} \sum_{j=1}^{N_{d}} \frac{\partial}{\partial \xi_{j}}\left(\alpha_{2} \rho_{2} U_{j}\right)=0, \\
& \frac{\partial}{\partial t}\left(\rho u_{i}\right)+\frac{1}{J} \sum_{j=1}^{N_{d}} \frac{\partial}{\partial \xi_{j}}\left(\rho u_{i} U_{j}+p J_{j i}\right)=0, \quad i=1,2, \ldots, N_{d}, \\
& \frac{\partial \alpha_{1}}{\partial t}+\frac{1}{J} \sum_{j=1}^{N_{d}} U_{j} \frac{\partial \alpha_{1}}{\partial \xi_{j}}=\mu\left(p_{1}\left(\rho_{1}\right)-p_{2}\left(\rho_{2}\right)\right),
\end{aligned}
$$

that is fundamental in our method on adaptive moving meshes. Here the quantities $J_{i j}$ for $i, j=1,2,3$ are as a result of the coordinate change that satisfies the following expressions:

$$
\left(\begin{array}{lll}
J_{11} & J_{12} & J_{13} \\
J_{21} & J_{22} & J_{23} \\
J_{31} & J_{32} & J_{33}
\end{array}\right)=\left(\begin{array}{lll}
b_{2} c_{3}-b_{3} c_{2} & a_{3} c_{2}-a_{2} c_{3} & a_{2} b_{3}-a_{3} b_{2} \\
b_{3} c_{1}-b_{1} c_{3} & a_{1} c_{3}-a_{3} c_{1} & a_{3} b_{1}-a_{1} b_{3} \\
b_{1} c_{2}-b_{2} c_{1} & a_{2} c_{1}-a_{1} c_{2} & a_{1} b_{2}-a_{2} b_{1}
\end{array}\right),
$$

and the quantity $J=\operatorname{det}\left|\partial\left(x_{1}, x_{2}, x_{3}\right) / \partial\left(\xi_{1}, \xi_{2}, \xi_{3}\right)\right|$ is the Jacobian of the mapping which can be computed by

$$
J=\sum_{i=1}^{3} a_{i} J_{1 i}=\sum_{i=1}^{3} b_{i} J_{2 i}=\sum_{i=1}^{3} c_{i} J_{3 i}
$$

Note that during the initialization step, all the coordinate transformation variables such as $a_{i}, b_{i}, c_{i}, J_{1 i}, J_{2 i}, J_{3 i}$ for $i=1,2,3$, 
and $J$ would be determined and remained fixed at all time when a mapped grid is constructed by a chosen numerical grid generator (cf. $[12,13])$.

It is easy to see that (5) would be a two-dimensional coordinate mapping from $\left(x_{1}, x_{2}\right)$ to $\left(\xi_{1}, \xi_{2}\right)$ for any spatial location $x_{3}$ in the physical domain, if we have a simplified data set where the quantities $a_{3}, b_{3}, c_{1}$, and $c_{2}$ are all zero, and $c_{3}$ is equal to one. In this instance, if we set $N_{d}=2$ in (6) with the coordinate transformation variables defined as in (7) and (8), we would have the same relaxation model in a two-dimensional curvilinear coordinate when a mapping of the form

$$
\begin{aligned}
& d x_{1}=a_{1} d \xi_{1}+a_{2} d \xi_{2}, \\
& d x_{2}=b_{1} d \xi_{1}+b_{2} d \xi_{2}
\end{aligned}
$$

is used in the derivation (cf. $[14,15])$. Thus, without causing any confusion, we may simply use the symbol $N_{d}$ as in the Cartesian case, to represent the number of spatial dimension in the curvilinear-coordinate formulation of equations.

For convenience, we write the relaxation model described above into a more compact expression by

$$
\frac{\partial q}{\partial t}+\frac{1}{J} \sum_{j=1}^{N_{d}}\left(\frac{\partial}{\partial \xi_{j}} f_{j}(q)+B_{j}(q) \frac{\partial q}{\partial \xi_{j}}\right)=\mu \psi(q),
$$

with $q, f_{j}, B_{j}$, and $\psi$ defined in turn by

$$
\begin{aligned}
q & =\left(\alpha_{1} \rho_{1}, \alpha_{2} \rho_{2}, \rho u_{1}, \ldots, \rho u_{N_{d}}, \alpha_{1}\right)^{T} \\
f_{j} & =\left(\alpha_{1} \rho_{1} U_{j}, \alpha_{2} \rho_{2} U_{j}, \rho u_{1} U_{j}+p J_{j 1}, \ldots, \rho u_{N_{d}} U_{j}+p J_{j, N_{d}}, 0\right)^{T} \\
B_{j} & =\operatorname{diag}\left(0, \ldots, 0, U_{j}\right) \\
\psi & =\left(0, \ldots, 0, p_{1}\left(\rho_{1}\right)-p_{2}\left(\rho_{2}\right)\right)^{T}
\end{aligned}
$$

Note that in the Cartesian coordinates case where the coordinate mapping quantities $a_{1}, b_{2}, c_{3}$ are all equal to one, while the remaining ones are all zeros, (10) reduces to

$$
\frac{\partial q}{\partial t}+\sum_{j=1}^{N_{d}}\left(\frac{\partial}{\partial x_{j}} \breve{f}_{j}(q)+\breve{B}_{j}(q) \frac{\partial q}{\partial x_{j}}\right)=\mu \psi(q)
$$

with

$$
\begin{aligned}
\breve{f}_{j} & =\left(\alpha_{1} \rho_{1} u_{j}, \alpha_{2} \rho_{2} u_{j}, \rho u_{1} u_{j}+p \delta_{1 j}, \ldots, \rho u_{N_{d}} u_{j}+p \delta_{3 j}, 0\right)^{T} \\
\breve{B}_{j} & =\operatorname{diag}\left(0, \ldots, 0, u_{j}\right)
\end{aligned}
$$

Then it is easy to check that $f_{j}$ and $B_{j}$ are related to $\breve{f}_{j}$ and $\breve{B}_{j}$ via $f_{j}=\sum_{i=1}^{N_{d}} \breve{f}_{i} J_{j i}$ and $B_{j}=\sum_{i=1}^{N_{d}} \breve{B}_{i} J_{j i}$, respectively.
With these notations, by assuming the proper smoothness of the solutions, the quasi-linear form of our model (10) can be written as

$$
\frac{\partial q}{\partial t}+\frac{1}{J} \sum_{j=1}^{N_{d}}\left(A_{j}(q)+B_{j}(q)\right) \frac{\partial q}{\partial \xi_{j}}=\mu \psi(q)
$$

where $A_{j}=\partial f_{j} / \partial q=\sum_{i=1}^{N_{d}} \breve{A}_{i} J_{j i}$ is the Jacobian matrix of $f_{j}$ with $\breve{A}_{i}=\partial \breve{f}_{i} / \partial q$ for $i=1,2, \ldots, N_{d}$. If we assume further that the thermodynamic description of the materials of interest is limited by the stability requirement, it is a straightforward matter to show that any linear combination of the matrices $\breve{A}_{i}+\breve{B}_{i}$ for $i=1,2, \ldots, N_{d}$ is diagonalizable with real eigenvalues and a complete set of linearly independent right eigenvectors (cf. [16]). Hence, we may conclude that this relaxation model is hyperbolic. Regarding discontinuous solutions of the system, such as shock waves or contact discontinuities, we find the usual form of the Rankine-Hugoniot jump conditions across the waves (cf. [17]).

\section{NUMERICAL METHODS ON MAPPED GRIDS}

To set the groundwork for the later development of an adaptive moving mesh method, we describe a finite volume method in wave-propagation form (cf. $[12,18,19])$ for the numerical approximation of our relaxation model (without the source terms)

$$
\frac{\partial q}{\partial t}+\frac{1}{J} \sum_{j=1}^{N_{d}}\left(\frac{\partial}{\partial \xi_{j}} f_{j}(q)+B_{j}(q) \frac{\partial q}{\partial \xi_{j}}\right)=0
$$

on a mapped grid. The method is based on solving onedimensional Riemann problems at each cell edge, and the waves (i.e., discontinuities moving at constant speeds) arising from the Riemann problem are employed to update the cell averages in the cells neighboring each edge.

To review the basic idea of the method, we consider the twodimensional $N_{d}=2$ quadrilateral grid case as illustrated in Fig. 1, for example. In a finite volume method, the approximate value of the cell average of the solution $q$ over the $(i, j)$ th grid cell at a time $t_{n}$ can be written as

$$
\begin{aligned}
Q_{i j}^{n} & \approx \frac{1}{\mathcal{M}\left(C_{i j}\right)} \int_{C_{i j}} q\left(x_{1}, x_{2}, t_{n}\right) d x_{1} d x_{2} \\
& =\frac{1}{\kappa_{i j} \Delta \xi_{1} \Delta \xi_{2}} \int_{\hat{C}_{i j}} q\left(\xi_{1}, \xi_{2}, t_{n}\right) d \xi_{1} d \xi_{2}
\end{aligned}
$$

where $\mathcal{C}_{i j}$ and $\hat{\mathcal{C}}_{i j}$ denote the regions occupied by the grid cell $(i, j)$ in the physical and computational domains, respectively, and $\mathcal{M}\left(C_{i j}\right)=\kappa_{i j} \Delta \xi_{1} \Delta \xi_{2}$ is the measure (area) of $\mathcal{C}_{i j}$. 


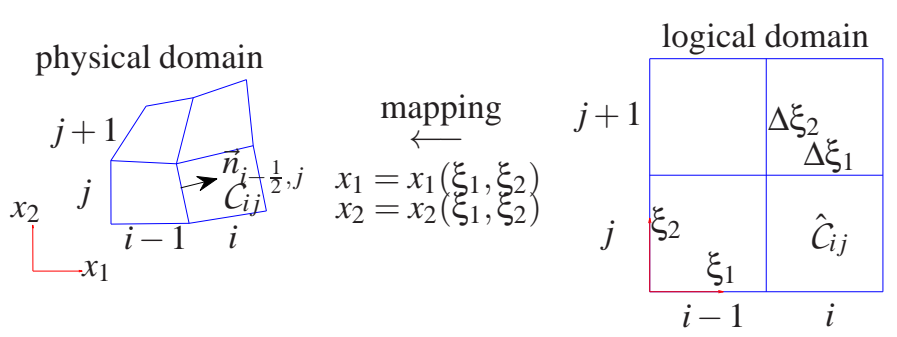

Figure 1. A SAMPLE QUADRILATERAL GRID IN OUR TWODIMENSIONAL NUMEIRCAL METHOD.

In this setup, a fully discrete version of the first-order wave propagation method for the equations (13) is a Godunov-type scheme on a quadrilateral grid that takes the form

$$
\begin{aligned}
& Q_{i j}^{n+1}=Q_{i j}^{n}-\frac{1}{\kappa_{i j}} \frac{\Delta t}{\Delta \xi_{1}}\left(\mathcal{A}_{1}^{+} \Delta Q_{i-\frac{1}{2}, j}+\mathcal{A}_{1}^{-} \Delta Q_{i+\frac{1}{2}, j}\right)- \\
& \frac{1}{\kappa_{i j}} \frac{\Delta t}{\Delta \xi_{2}}\left(\mathcal{A}_{2}^{+} \Delta Q_{i, j-\frac{1}{2}}+\mathcal{A}_{2}^{-} \Delta Q_{i, j+\frac{1}{2}}\right) .
\end{aligned}
$$

Here $\mathcal{A}_{1}^{+} \Delta Q_{i-\frac{1}{2}, j}, \mathcal{A}_{1}^{-} \Delta Q_{i+\frac{1}{2}, j}, \mathcal{A}_{2}^{+} \Delta Q_{i, j-\frac{1}{2}}$, and $\mathcal{A}_{2}^{-} \Delta Q_{i, j+\frac{1}{2}}$ are the right-, left-, up-, and down-moving fluctuations, respectively, that are entering into the grid cell. To determine these fluctuations, we need to solve the one-dimensional Riemann problems normal to the cell edges.

\section{Computing Fluctuations}

Considering the fluctuations $\mathcal{A}_{1}^{ \pm} \Delta Q_{i-\frac{1}{2}, j}$ arising from the edge $\left(i-\frac{1}{2}, j\right)$ between cells $(i-1, j)$ and $(i, j)$, for example. This amounts to solve a Cauchy problem in the $\xi_{1}$-direction that consists of

$$
\frac{\partial q}{\partial t}+\frac{1}{J} \frac{\partial f_{1}(q)}{\partial \xi_{1}}+B_{1}(q) \frac{1}{J} \frac{\partial q}{\partial \xi_{1}}=0
$$

as for the equations and the piecewise constant data

$$
q\left(\xi_{1}, \xi_{2}, t_{n}\right)= \begin{cases}Q_{i-1, j}^{n} & \text { if } \xi_{1}<\left(\xi_{1}\right)_{i-\frac{1}{2}} \\ Q_{i j}^{n} & \text { if } \xi_{1}>\left(\xi_{1}\right)_{i-\frac{1}{2}}\end{cases}
$$

as for the initial condition at a time $t_{n}$.

Let $\vec{n}_{i-\frac{1}{2}, j}=\left(\hat{b}_{2},-\hat{a}_{2}\right)_{i-\frac{1}{2}, j}$ and $\vec{t}_{i-\frac{1}{2}, j}=\left(\hat{a}_{2}, \hat{b}_{2}\right)_{i-\frac{1}{2}, j}$ be the unit normal and tangential vectors to the cell edge $\left(i-\frac{1}{2}, j\right)$ in the physical grid, where $\hat{a}_{i}=a_{i} / S_{i}$ and $\hat{b}_{i}=b_{i} / S_{i}$ are the scaled version of the metric elements $a_{i}$ and $b_{i}$ in (9) with $S_{i}=\sqrt{a_{i}^{2}+b_{i}^{2}}$ for $i=1,2$. Then to compute $\mathcal{A}_{1}^{ \pm} \Delta Q_{i-\frac{1}{2}, j}$, as in $[12,18]$, we perform the following steps:
(1) Transform the data $Q_{i-1, j}^{n}$ and $Q_{i, j}^{n}$ into the new data $\breve{Q}_{L}=$ $\mathcal{R}_{i-\frac{1}{2}, j} Q_{i-1, j}^{n}$ and $\breve{Q}_{R}=\mathcal{R}_{i-\frac{1}{2}, j} Q_{i, j}^{n}$. Here $\mathcal{R}_{i-\frac{1}{2}, j}$ is a rotation matrix defined by

$$
\mathcal{R}_{i-\frac{1}{2}, j}=\left(\begin{array}{ccccc}
1 & 0 & 0 & 0 & 0 \\
0 & 1 & 0 & 0 & 0 \\
0 & 0 & \left(\hat{b}_{2}\right)_{i-\frac{1}{2}, j} & -\left(\hat{a}_{2}\right)_{i-\frac{1}{2}, j} & 0 \\
0 & 0 & \left(\hat{a}_{2}\right)_{i-\frac{1}{2}, j} & \left(\hat{b}_{2}\right)_{i-\frac{1}{2}, j} & 0 \\
0 & 0 & 0 & 0 & 1
\end{array}\right) .
$$

(2) Solve Riemann problem in the " $x_{1}$ " direction for

$$
\frac{\partial q}{\partial t}+\frac{\partial}{\partial x_{1}} \breve{f}_{1}(q)+\breve{B}_{1}(q) \frac{\partial q}{\partial x_{1}}=0
$$

with $\breve{f}_{1}$ and $\breve{B}_{1}$ defined by (11) and the Riemann data $\breve{Q}_{L}$ and $\breve{Q}_{R}$. When an approximate Riemann solver is used for the numerical resolution, this would result in propagating discontinuities that are moving with speeds $\breve{\lambda}_{i-\frac{1}{2}, j}^{1, m}$ and the jumps $\breve{\mathcal{W}}_{i-\frac{1}{2}, j}^{1, m}$ across each of them for $m=1,2, \ldots, M_{w}$, see $[8,16]$ for an example.

(3) Define scaled speeds $\lambda_{i-\frac{1}{2}, j}^{1, m}=\left(S_{2}\right)_{i-\frac{1}{2}, j} \breve{\lambda}_{i-\frac{1}{2}, j}^{1, m}$ and rotate jumps back to the Cartesian coordinates by $\mathcal{W}_{i-\frac{1}{2}, j}^{1, m}=$ $\mathcal{R}_{i-\frac{1}{2}, j}^{T} \breve{W}_{i-\frac{1}{2}, j}^{1, m}$ for $m=1,2, \ldots, M_{w}$.

(4) Determine the left- and right-moving fluctuations as

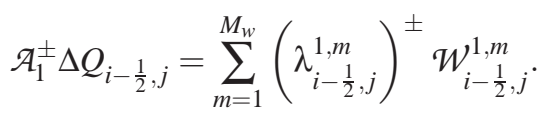

As usual, the notations for the quantities $\lambda^{ \pm}$are set by $\lambda^{+}=$ $\max (\lambda, 0)$ and $\lambda^{-}=\min (\lambda, 0)$.

In a similar manner, we may determine the up- and downmoving fluctuations at the edge $\left(i, j-\frac{1}{2}\right)$ in the form

$$
\mathcal{A}_{2}^{ \pm} \Delta Q_{i, j-\frac{1}{2}}=\sum_{m=1}^{M_{w}}\left(\lambda_{i, j-\frac{1}{2}}^{2, m}\right)^{ \pm} \mathcal{W}_{i, j-\frac{1}{2}}^{2, m}
$$

that is as the result of solving

$$
\frac{\partial q}{\partial t}+\frac{1}{J} \frac{\partial f_{2}(q)}{\partial \xi_{2}}+B_{2}(q) \frac{1}{J} \frac{\partial q}{\partial \xi_{2}}=0
$$

with the initial data $Q_{i, j-1}^{n}$ and $Q_{i, j}^{n}$. 


\section{High-Resolution Correction}

To achieve high resolution (i.e., second-order accurate on smooth solutions, and sharp and monotone profiles on discontinuous solutions), the speeds and the limited version of the jumps are used to construct the piecewise linear correction terms as before (cf. [18]), and are added to (14) in flux difference form as

$$
\begin{aligned}
& Q_{i j}^{n+1}:=Q_{i j}^{n+1}-\frac{1}{\kappa_{i j}} \frac{\Delta t}{\Delta \xi_{1}}\left(\widetilde{\mathcal{F}}_{i+\frac{1}{2}, j}^{1}-\widetilde{\mathcal{F}}_{i-\frac{1}{2}, j}^{1}\right)- \\
& \frac{1}{\kappa_{i j}} \frac{\Delta t}{\Delta \xi_{2}}\left(\widetilde{\mathcal{F}}_{i, j+\frac{1}{2}}^{2}-\widetilde{\mathcal{F}}_{i, j-\frac{1}{2}}^{2}\right) .
\end{aligned}
$$

Here at the edge $\left(i-\frac{1}{2}, j\right)$ the correction flux takes the form

$$
\widetilde{\mathcal{F}}_{i-\frac{1}{2}, j}^{1}=\frac{1}{2} \sum_{m=1}^{M_{w}}\left|\lambda_{i-\frac{1}{2}, j}^{1, m}\right|\left(1-\frac{\Delta t}{\kappa_{i-\frac{1}{2}, j} \Delta \xi_{1}}\left|\lambda_{i-\frac{1}{2}, j}^{1, m}\right|\right) \widetilde{\mathcal{W}}_{i-\frac{1}{2}, j}^{1, m},
$$

where $\kappa_{i-\frac{1}{2}, j}=\left(\kappa_{i-1, j}+\kappa_{i, j}\right) / 2$. The quantity $\widetilde{\mathcal{W}}^{1, m}$ is a limited value of $\mathcal{W}^{1, m}$ obtained by comparing $\mathcal{W}^{1, m}$ with the corresponding $\mathcal{W}^{1, m}$ from the neighboring Riemann problem to the left (if $\lambda^{1, m}>0$ ) or to the right (if $\lambda^{1, m}<0$ ) for $m=1,2, \ldots, M_{w}$. The correction flux $\tilde{\mathcal{F}}_{i, j-\frac{1}{2}}^{2}$ at the edge $\left(i, j-\frac{1}{2}\right)$ can be defined in a similar manner.

In addition to that, a transverse propagation of wave is also included in the method as a part of the high-resolution correction terms, see [18] for the details. With the transverse wave propagation, the method is typically stable as long as the time step $\Delta t$ satisfies a variant of the CFL (Courant-Friedrichs-Lewy) condition of the form

$$
v=\Delta t \max _{i, j, m}\left(\frac{\left|\lambda_{i-\frac{1}{2}, j}^{1, m}\right|}{\kappa_{i_{p}, j} \Delta \xi_{1}}, \frac{\left|\lambda_{i, j-\frac{1}{2}}^{2, m}\right|}{\kappa_{i, j_{p}} \Delta \xi_{2}}\right) \leq 1,
$$

where $i_{p}=i$ if $\lambda_{i-\frac{1}{2}, j}^{1, m}>0$ and $i-1$ if $\lambda_{i-\frac{1}{2}, j}^{1, m}<0 ; j_{p}$ is defined analogously.

\section{RELAXATION SOLVER ON MOVING MESHES}

One simple way to extend the above mapped grid method to a moving grid version is to take an interpolation-based approach proposed by Tang and Tang [20]. In the current case with the relaxation model (10), in each time step, our method consists of the following steps:
(1) (Mesh redistribution step) Solve an elliptic-type meshredistribution equation for the new location of the mesh vertices, and then interpolate the numerical solution on the resulting grid conservatively. This step should be done in an iterative manner until convergence.

(2) (Zero relaxation step) Solve the homogeneous part of the relaxation model (10) on a new grid obtained in step 1.

(3) (Pressure relaxation step) Solve the model system with only the source terms in the infinite relaxation limit, yielding the relaxed volume fraction and so the equilibrium pressure.

Since the numerical method for step 2 of the algorithm has been described before, we now focus our discussion on steps 1 and 3 below.

\section{Mesh Redistribution Scheme}

In the interpolation-based moving mesh method considered here, it is a common approach to redistribute the mesh vertices $x_{i}$ for $i=1,2, \ldots, N_{d}$ based on the solution of the elliptic-type equation of the form

$$
\sum_{j=1}^{N_{d}} \frac{\partial}{\partial \xi_{j}}\left(D(q) \frac{\partial x_{i}}{\partial \xi_{j}}\right)=0
$$

with prescribed boundary conditions on the domain. Note that (17) are the Euler-Lagrange equations associated with a variational problem for equidistribution adaptive meshes (cf. [21]). Here $D$ is called a monitor function that is chosen to measure the degree of regularity of the underlying solution. In this work, it is taken as

$$
D(q)=\frac{(1-\beta)|\nabla q|+\beta\|\nabla q\|_{1}}{(1-\beta)\|\nabla q\|_{\infty}+\beta\|\nabla q\|_{1}}
$$

where $\beta \in[0,1]$ is a free parameter, see [22] for more details.

Consider the two-dimensional case $N_{d}=2$ as an example, we may discretize (17) using a standard 5-point stencil finitedifference formula

$$
\begin{gathered}
\frac{1}{\Delta \xi_{1}^{2}}\left(D_{i-\frac{1}{2}, j}^{k} Z_{i-1, j}^{k}-\left(D_{i-\frac{1}{2}, j}^{k}+D_{i+\frac{1}{2}, j}^{k}\right) Z_{i j}^{k}+D_{i+\frac{1}{2}, j}^{k} Z_{i+1, j}^{k}\right)+ \\
\frac{1}{\Delta \xi_{2}^{2}}\left(D_{i, j-\frac{1}{2}}^{k} Z_{i, j-1}^{k}-\left(D_{i, j-\frac{1}{2}}^{k}+D_{i, j+\frac{1}{2}}^{k}\right) Z_{i j}^{k}+D_{i, j+\frac{1}{2}}^{k} Z_{i, j+1}^{k}\right)=0,
\end{gathered}
$$

where $Z^{k}$ represents the $k$ th iterate of the computed mesh vertices for $x_{1}$ and $x_{2}$. The resulting linear system can be solved by a Gauss-Seidel type iteration, see Fig. 2 for an illustration. 


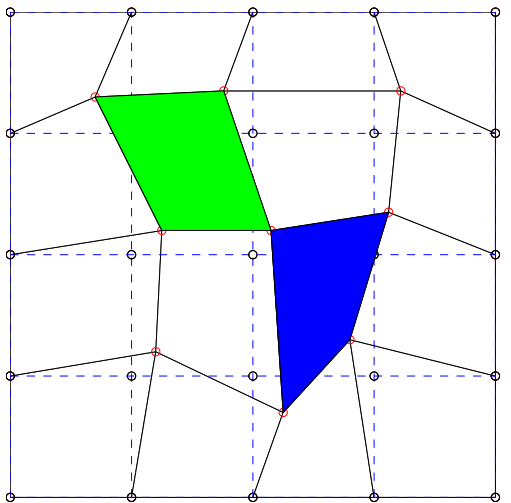

Figure 2. A SAMPLE NEW MESH (GRAPHED AS SOLID LINES) AFTER MESH REDISTRIBUTION OF INITIAL MESH (DASHED LINES). THE NUMERICAL SOLUTIONS IN SHADED REGION OF THE NEW MESH, FOR EXAMPLE, SHOULD BE UPDATED CONSERVATIVELY.

We note that after each mesh redistribution iterate $k$, the numerical solutions on this mesh should be interpolated conservatively, i.e.,

$$
\sum_{\forall s} \mathcal{M}\left(C_{s}^{k+1}\right) Q_{s}^{k+1}=\sum_{\forall s} \mathcal{M}\left(C_{s}^{k}\right) Q_{s}^{k}
$$

This can be done quite easily by using either a finite-volume or a geometric approach (cf. [9]).

It should be mentioned also that rather than using the above iterative procedure for mesh redistribution, for some applications such as problems with free-surface or moving boundary, it may be more efficient to use a non-iterative Lagrangian approach (cf. [23]) instead over a prescribed time step $\Delta t$; this can be done at the least before the time when grid tangling occurs (cf. [24]).

\section{Pressure Relaxation}

Finally, in step 3, using the solution obtained in step 2 as the initial condition, we solve the model system with the source term of the form

$$
\frac{\partial q}{\partial t}=\mu \psi(q)
$$

which, in the infinite relaxation limit, leads to

$$
\hat{g}\left(\alpha_{1}^{n+1}\right)=p_{1}\left(\frac{\left(\alpha_{1} \rho_{1}\right)^{n+1}}{\alpha_{1}^{n+1}}\right)-p_{2}\left(\frac{\left(\alpha_{2} \rho_{2}\right)^{n+1}}{1-\alpha_{1}^{n+1}}\right)=0
$$

for the relaxed volume fraction $\alpha_{1}^{n+1}$ which can be solved by a standard iterative root-finding solver such as the secant method.

\section{NUMERICAL RESULTS}

We now present numerical results obtained using our moving mesh method for two-phase barotropic flow problems with and without cavitation. For simplicity, we assume that the constitutive law for each of the fluid phases satisfies the Tait equation of state of the form

$$
p_{k}(\rho)=\left(p_{0 k}+\mathcal{B}_{k}\right)\left(\frac{\rho}{\rho_{0 k}}\right)^{\gamma_{k}}-\mathcal{B}_{k}
$$

where $p_{0 k}$ and $\rho_{0 k}$ are the pressure and density at a reference state, $\gamma_{k}$ is the ratio of specific heats, and $\mathcal{B}_{k}$ is a pressure-like constant for $k=1,2$. The set of parameters we take throughout the tests are, for phase 1 (the liquid phase), $\left(\gamma, \mathcal{B}, \rho_{0}, p_{0}\right)_{1}=$ $\left(7,3000 \mathrm{bar}, 10^{3} \mathrm{~kg} / \mathrm{m}^{3}, 1 \mathrm{bar}\right)$, and for phase 2 (the gas phase), $\left(\gamma, \mathcal{B}, \rho_{0}, p_{0}\right)_{2}=\left(1.4,0,1 \mathrm{~kg} / \mathrm{m}^{3}, 1 \mathrm{bar}\right)$.

\section{One-Dimensional Water-Vapor Cavitation}

Our first example is a water-vapor cavitation problem in that inside a shock tube of one-meter length with closed ends the fluid is a homogeneous air-water mixture at the standard atmospheric condition $\left(\rho_{1}, \rho_{2}, \alpha_{1}\right)=\left(10^{3} \mathrm{~kg} / \mathrm{m}^{3}, 1 \mathrm{~kg} / \mathrm{m}^{3}, 1-\varepsilon\right)$, with $\varepsilon=$ $10^{-2}$. Initially, inside the tube, there is a jump on the velocity at $x_{1}=1 / 2 \mathrm{~m}$ with speed $u_{1}=-100 \mathrm{~m} / \mathrm{s}$ on the left and $u_{1}=$ $100 \mathrm{~m} / \mathrm{s}$ on the right of the tube. In addition to that, since it is a closed tube, there are jumps also on the velocity at the both ends.

With this condition, as times go on, two rarefaction waves are formed, causing the decrease of the pressure and the formation of the cavitation zone inside the tube. In the meantime, there are inward-moving shock waves propagating from the boundaries, yielding the collapse of the region of cavitation due to the shock-cavitation interaction. Figure 3 shows contours of the volume fraction and pressure in the $x_{1}-t$ plane up to time $0.8 \mathrm{~ms}$, see Fig. 4 for the meshes over time. We observe reasonable resolution of the results as compared to the ones shown in [6].

\section{Two-Dimensional Underwater Explosion}

Our second example concerns with a model two-phase underwater explosion problem (cf. [25]). In this test, we take a rectangular domain $\left(x_{1}, x_{2}\right) \in[-2,2] \times[-1.5,1] \mathrm{m}^{2}$, and employ the initial condition that consists of a stationary horizontal free surface at the $x_{2}=0$ axis and a circular gas bubble in water with the center $\left(x_{1}^{0}, x_{2}^{0}\right)=(0,-0.3) \mathrm{m}$ and of the radius $r_{0}=0.12 \mathrm{~m}$. Here above the free surface, the fluid is the gas phase at the standard atmospheric condition $\left(\rho_{1}, \rho_{2}, \alpha_{1}\right)=$ $\left(1 \mathrm{~kg} / \mathrm{m}^{3}, 10^{3} \mathrm{~kg} / \mathrm{m}^{3}, 1-\varepsilon\right)$. with $\varepsilon=10^{-6}$. Below the free surface, in region inside the gas bubble the fluid is modeled as a perfect gas also with the state variables $\left(\rho_{1}, \rho_{2}, \alpha_{1}\right)=$ $\left(719.686 \mathrm{~kg} / \mathrm{m}^{3}, 10^{3} \mathrm{~kg} / \mathrm{m}^{3}, 1-\varepsilon\right)$, and in region outside the gas bubble the fluid is water with the state variables $\left(\rho_{1}, \rho_{2}, \alpha_{1}\right)=$ 

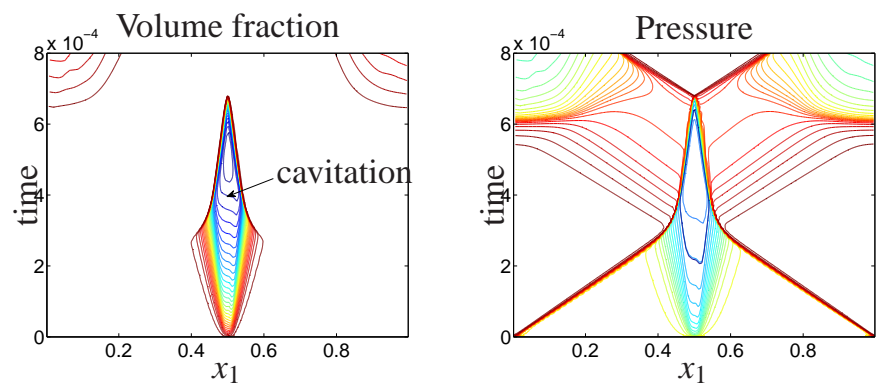

Figure 3. MOVING MESH RESULTS FOR A ONE-DIMENSIONAL CAVITATION PROBLEM. CONTOUR PLOTS OF THE VOLUME FRACTION AND PRESSURE ARE SHOWN IN THE $x_{1}$ - $t$ PLANE UP TO $0.8 \mathrm{~ms}$.

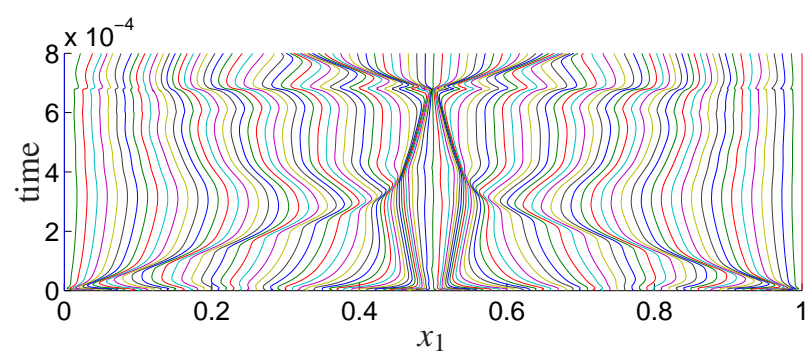

Figure 4. MOVING MESHES FOR THE RESULTS SHOWN IN FIG. 3.

$\left(1 \mathrm{~kg} / \mathrm{m}^{3}, 10^{3} \mathrm{~kg} / \mathrm{m}^{3}, \varepsilon\right)$, Here we take $\varepsilon=10^{-6}$. The boundary conditions considered here are solid wall on the left, right, and bottom sides, and non-reflecting on the top side of the domain.

Note that due to the pressure difference between the fluids at $r=r_{0}$, breaking of this underwater bubble would results in an outward-going shock wave in water, an inward-going rarefaction wave in gas, and a material interface lying in between that separates the gas and water. Soon after this shock wave is diffracted through the nearby flat air-water interface, it is known in the literature (cf. [25]) that the topology of the underwater bubble will undergo a change from the original circular-shape to an oval-like shape. As time evolves, this gas bubble would continue rising upward, causing the subsequent deformation of the horizontal air-water interface.

Contour plots of the density and pressure at four different times $t=0.2,0.4,0.8$, and $1.2 \mathrm{~ms}$ are presented in Fig. 5, where we have performed the computation using with a $200 \times 125$ grid. From the density plot, we observe clearly the basic feature of the solution structure as mentioned above, and from the pressure plot, we see the smooth variation of the solution near the interface, without introducing any spurious oscillations. Interestingly, these barotropic flow results are qualitatively similar to the nonbarotropic results as shown earlier in [24], for example. In Fig. 6, we present the mesh system for the run shown in Fig. 5; the dynamical movement of the grids is clearly seen.
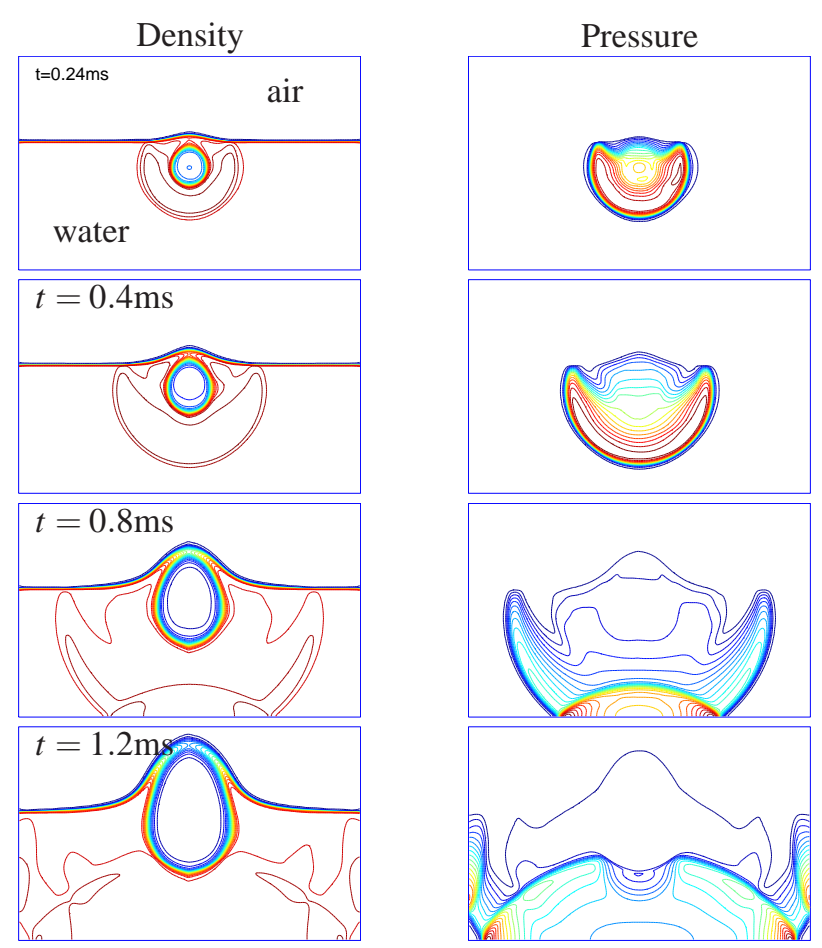

Figure 5. MOVING MESH RESULTS FOR A MODEL UNDERWATER EXPLOSION PROBLEM. DENSITY AND PRESSURE CONTOURS ARE SHOWN AT FOUR DIFFERENT TIMES $t=0.2,0.4,0.8$, and $1.2 \mathrm{~ms}$ OBTAINED USING A $200 \times 125$ GRID.
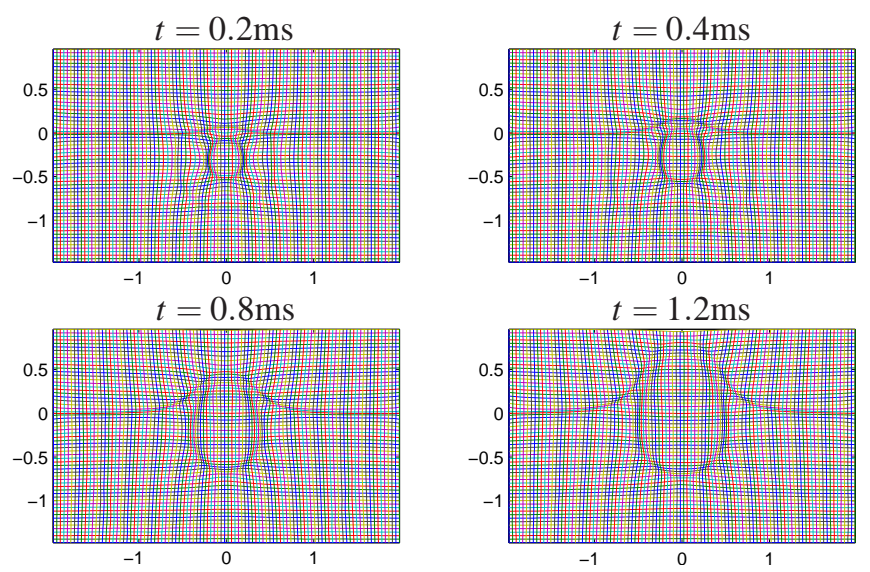

Figure 6. MOVING MESHES FOR THE RESULTS SHOWN IN FIG. 5.

\section{Two-Dimensional Supersonic Flow Over Bluntbody}

Our final example is a steady-state calculation of a supersonic flow over a bluntbody. Initially, the fluid is a homogeneous air-water mixture at the standard atmospheric condition $\left(\rho_{1}, \rho_{2}, \alpha_{1}\right)=\left(10^{3} \mathrm{~kg} / \mathrm{m}^{3}, 1 \mathrm{~kg} / \mathrm{m}^{3}, 1-\varepsilon\right)$, with $\varepsilon=10^{-2}$ in the whole domain. Supersonic air-water mixture is flowing into the domain with speed $u_{1}=600 \mathrm{~m} / \mathrm{s}$ from the left to right. Numerical results using a fixed $60 \times 48$ body-fitted grid is used in the com- 

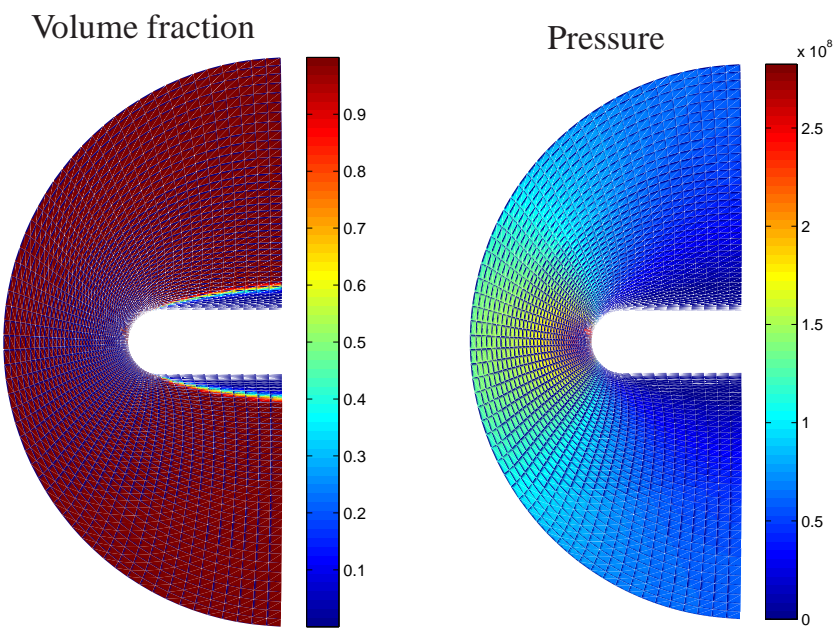

Figure 7. STEADY-STATE RESULTS FOR A TWO-DIMENSIONAL SUPERSONIC FLOW OVER A BLUNTBODY. PSEUDO COLORS OF THE VOLUME FRACTION AND PRESSURE ARE SHOWN.

putation. A cavitation zone near the top and bottom edge of the bluntbody is clearly seen. Note that there is a smooth transition in the pressure across the cavitation boundary without any spurious oscillations. Results obtained using a moving mesh version of the code will be reported elsewhere in that a suitable boundary redistribution scheme needs to be devised for the mesh adapatation near a general non-rectangular boundary, see [26,27] for a possible approach.

\section{SUMMARY}

We have presented a simple interpolation-based adaptive moving mesh method for the efficient numerical computation of compressible two-phase barotropic flow with and without cavitation. The algorithm uses a curvilinear-coordinate formulation of the relaxation model as the basis, and employs a wavepropagation based relaxed scheme to solve the model system on a mapped grid that is constructed by a conventional mesh redistribution procedure based on equidistribution principle. Numerical results for water-vapor cavitation in one dimension, underwater explosion in two dimensions, and steady state two-dimensional supersonic flow over bluntbody are present. Ongoing works are to extend this approach further to problems with phase transitions for general non-barotropic multiphase flow.

\section{ACKNOWLEDGMENT}

The author was supported in part by National Science Council of Taiwan Grants \#96-2115-M-002-008-MY3 and 99-2115M-002-005-MY2.

\section{REFERENCES}

[1] Franc, J.-P., and Michel, J.-M., 2005. Fundamentals of Cavitation. Dordrecht: Springer Science+Business Media.

[2] Brennen, C. E., 2005. Fundamentals of Multiphase Flow. Cambridge University Press.

[3] Young, F. R., 1989. Cavitation. McGraw-Hill.

[4] Venkateswaran, S., Lindau, J. W., Kunz, R. F., and Merkle, C. L., 2002. "Computation of multiphase mixture flows with compressibility effects”. J. Comput. Phys., 180, pp. 54-77.

[5] Saurel, R., Petitpas, F., and Berry, R. A., 2009. "Simple and efficient relaxation methods for interfaces separating compressible fluids, cavitating flows and shocks in multiphase mixtures". J. Comput. Phys., 228, pp. 1678-1712.

[6] Liu, T. G., Khoo, B. C., and Xie, W. F., 2004. "Isentropic one-fluid modelling of unsteady cavitating flow". J. Comput. Phys., 201, pp. 80-108.

[7] D.P. Schmidt, C.J. Rutland, M. C., 1999. "A fully compressible, two-dimensional model of small, high speed, cavitating nozzles". Atomization Sprays, 9, pp. 255-276.

[8] Shyue, K.-M., 2004. "A fluid-mixture type algorithm for barotropic two-fluid flow problems". J. Comput. Phys., 200, pp. 718-748.

[9] Tang, T., 2005. "Moving mesh methods for computational fluid dynamics". In Contemporary Mathematics, Vol. 383, Z.-C. Shi, Z. Chen, T. Tang, and D. Yu, eds., American Mathematical Society, Rhode Island, pp. 620-625.

[10] Petitpas, F., Massoni, J., Saurel, R., Lapebie, E., and Munier, L., 2009. "Diffuse interface model for high speed cavitating underwater systems". Int. J. Multiphase Flow, 35, pp. 747-759.

[11] Saurel, R., Petitpas, F., and Abgrall, R., 2008. "Modelling phase transition in metastable liquids: application to cavitating and flashing flows". J. Fluid. Mech., 607, pp. 313350 .

[12] Calhoun, D. A., Helzel, C., and LeVeque, R. J., 2008. "Logically rectangular grids and finite volume methods for PDEs in circular and spherical domains". SIAM Review, 50(4), pp. 723-752. Webpage to accompany this paper: http://www.amath.washington.edu/ rjl/pubs/circles/index.html

[13] Thompson, J. F., Soni, B. K., and Weatherill, N. P., 1999. Handbook of Grid Generation. CRC Press.

[14] Hoffmann, K. A., and Chiang, S. T., 2000. Computational Fluid Dynamics, 4 ed. Engineering Education System, Wichita, Kansas, USA.

[15] Wesseling, P., 2001. Principles of Computational Fluid Dynamics. Springer-Verlag.

[16] Allaire, G., Clerc, S., and Kokh, S., 2002. "A five-equation model for the simulation of interface between compressible fluids”. J. Comput. Phys., 181, pp. 577-616.

[17] Courant, R., and Friedrichs, K. O., 1948. Supersonic Flow and Shock waves. Wiley-Interscience, New York. 
[18] LeVeque, R. J., 2002. Finite Volume Methods for Hyperbolic Problems. Cambridge University Press.

[19] Shyue, K.-M., 2010. "A high-resolution mapped grid algorithm for compressible multiphase flow problems". $J$. Comput. Phys., 229, pp. 8780-8801.

[20] Tang, H., and Tang, T., 2003. "Adaptive mesh methods for one- and two-dimensional hyperbolic conservation laws". SIAM J. Numer. Anal., 41, pp. 487-515.

[21] Ceniceros, H. D., and Hou, T. Y., 2001. "An efficient dynamically adaptive mesh for potentially singular solutions". J. Comput. Phys., 172, pp. 609-639.

[22] van Dam, A., and Zegeling, P. A., 2010. "Balanced monitoring of flow phenomena in moving mesh methods". Comm. Comput. Phys., 7, pp. 138-170.

[23] Addessio, F. L., Baumgardner, J. R., Dukowicz, J. K., Johnson, N. L., Kashiwa, B. A., Rauenzahn, R. M., and Zemach, C., 1990. "CAVEAT: A computer code for fluid dynamics problems with large distortion and internal slip". $L A$ 10613-MS-REV. 1.

[24] Shyue, K.-M., 2009. "A simple unified coordinates method for compressible homogeneous two-phase flows". In Proc. Symp. Appl. Math., Vol. 67, E. Tadmor, J.-G. Liu, and A. Tzavaras, eds., American Mathematical Society, pp. 949-958.

[25] Grove, J., and Menikoff, R., 1990. "The anomalous reflection of a shock wave at a material interface". J. Fluid Mech., 219, pp. 313-336.

[26] Li, R., Tang, T., and Zhang, P., 2001. "Moving mesh mehods in multiple dimensions based on harmonic maps". J. Comput. Phys., 170, pp. 562-588.

[27] Tang, H. Z., 2006. "A moving mesh method for the Euler calculations using a directional monitor function". Comm. Comput. Phys., 1, pp. 656-676. 